\title{
Saponin-Rich Extracts and Their Acid Hydrolysates Differentially Target Colorectal Cancer Metabolism in the Frame of Precision Nutrition
}

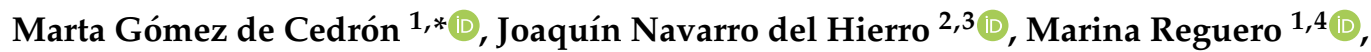 \\ Sonia Wagner ${ }^{1,5,+}$, Adrián Bouzas ${ }^{1,6,+}$, Adriana Quijada-Freire ${ }^{1}$, Guillermo Reglero ${ }^{2,3}(\mathbb{0}$, \\ Diana Martín 2,3 (D) and Ana Ramírez de Molina ${ }^{1, *(\mathbb{D})}$ \\ 1 Precision Nutrition and Cancer Program, Molecular Oncology Group, IMDEA Food Institute, \\ CEI UAM + CSIC, E-28049 Madrid, Spain; marina.reguero@imdea.org (M.R.); \\ sonia.wagner@imdea.org (S.W.); adrian.bouzas@imdea.org (A.B.); Adriana.quijada@imdea.org (A.Q.-F.) \\ 2 Department of Production and Characterization of Novel Foods, Institute of Food Science \\ Research (CIAL) (CSIC.UAM), 28049 Madrid, Spain; joaquin.navarrodel@uam.es (J.N.d.H.); \\ guillermo.reglero@uam.es (G.R.); diana.martin@uam.es (D.M.) \\ 3 Sección Departamental de Ciencias de la Alimentación, \\ Facultad de Ciencias, Universidad Autónoma de Madrid, 28049 Madrid, Spain \\ 4 NATAC BIOTECH, Electronica 7, 28923 Madrid, Spain \\ 5 Medicinal Gardens SL, Marques de Urquijo 47, 28008 Madrid, Spain \\ 6 Forchronic, CANAAN Research \& Investment Group, Agustín de Betancourt 21, 28003 Madrid, Spain \\ * Correspondence: marta.gomezdecedron@imdea.org (M.G.d.C.); ana.ramirez@imdea.org (A.R.d.M.) \\ + These authors contribute equally.
}

Received: 20 October 2020; Accepted: 14 November 2020; Published: 17 November 2020

Simple Summary: Colorectal cancer remains the second leading cause of cancer-related death worldwide, which is a situation that requires the continuous search for natural bioactive compounds that can mitigate its effects or can be used as co-adjuvants in the frame of precision nutrition. The elucidation of the mechanisms underlying the bioenergetics of colorectal tumorous cells as well as the modulation of lipid metabolism-related genes may greatly contribute to personalized therapies. We show that saponin- and sapogenin-rich extracts from fenugreek are able to strongly inhibit colorectal cancer cells' growth, which is related to a reduction in the mitochondrial oxidative phosphorylation and the inhibition of aerobic glycolysis, in which each of the extracts target the molecular pathways related to lipid metabolism in a different manner. We propose that this outcome may have important implications on their potential use against colon cancer in the context of precision nutrition.

\begin{abstract}
Saponins or their aglycone form, sapogenin, have recently gained interest as bioactive agents due to their biological activities, their antitumoral effects being among them. Metabolic reprogramming has been recognized as a hallmark of cancer and, together with the increased aerobic glycolysis and glutaminolysis, the altered lipid metabolism is considered crucial to support cancer initiation and progression. The purpose of this study was to assess and compare the inhibitory effects on colorectal cancer cell lines of saponin-rich extracts from fenugreek and quinoa (FE and QE, respectively) and their hydrolyzed extracts as sapogenin-rich extracts (HFE and HQE, respectively). By mean of the latest technology in the analysis of cell bioenergetics, we demonstrate that FE and HFE diminished mitochondrial oxidative phosphorylation and aerobic glycolysis; meanwhile, quinoa extracts did not show relevant activities. Distinct molecular mechanisms were identified for fenugreek: FE inhibited the expression of TYMS1 and TK1, synergizing with the chemotherapeutic drug 5-fluorouracil (5-FU); meanwhile, HFE inhibited lipid metabolism targets, leading to diminished intracellular lipid content. The relevance of considering the coexisting compounds of the extracts or their hydrolysis
\end{abstract}


transformation as innovative strategies to augment the therapeutic potential of the extracts, and the specific subgroup of patients where each extract would be more beneficial, are discussed in the frame of precision nutrition.

Keywords: fenugreek; quinoa; saponins; sapogenins; colorectal cancer; metabolic reprogramming; cell bioenergetics; precision nutrition

\section{Introduction}

Cancer is a complex and heterogeneous disease where genetic and environmental factors contribute to initiation, progression, and resistance to treatments. Metabolic reprogramming has been recognized as a hallmark of cancer [1,2]. Tumor cells frequently upregulate aerobic glycolysis (Warburg effect) and glutaminolysis to sustain cell proliferation. However, in recent years, alterations in lipid metabolism have been identified as key factors in cancer [2-4]. Lipids represent an important source of energy, as well as structural resources for the tumor, and they are also important signaling molecules for cellular pathways and intercellular communication. Although genetic alterations are the most studied factors, environmental factors including those related to lifestyle also contribute to the onset and progression of cancer. The World Health Organization (WHO) estimates that up to one-third of all cancers could be prevented through lifestyle changes, such as diet. In the case of gastric tumors, this percentage rises to more than half, constituting the main factor in their development [5]. Colorectal cancer (CRC) is the second most prevalent type of cancer in the world, and its appearance is closely associated to obesity and metabolic syndrome.

The recent development of powerful "omics" technologies (genomics, transcriptomics, proteomics, metabolomics, and lipidomics) has opened new avenues in nutritional sciences toward precision nutrition, which takes into account the differential responses to nutritional interventions based on gene variation (nutrigenetics), together with the scientific knowledge of the molecular targets and mechanism of action of bioactive compounds present in the diet (nutrigenomics). Moreover, precision nutrition also takes into account the nutritional and metabolic status of the individuals-obesity, T2DM (Type 2 Diabetes Mellitus), metabolic syndrome, mainly due to its association to chronic low-grade inflammation; life style parameters-alcohol consumption, diet, exercise; and the intestinal microbiome [6]. Precision nutrition can be considered as a therapeutic tool against chronic diseases related to metabolism, including cancer. This way, diet-derived ingredients, bioactive compounds from natural sources, and nutritional strategies can be developed as co-adjuvants in combination with the clinical therapies in cancer treatment $[7,8]$.

Plants and food-derived ingredients constitute an inexhaustible source of bioactive compounds that may effectively inhibit the growth and progression of cancer cells and/or synergize with chemotherapeutic drugs used in the clinics, with fewer adverse and toxic effects. The success of the use of bioactive compounds in precision nutrition as co-adjuvants in the treatment of cancer patients requires the knowledge of their molecular targets and mechanism of action, which will identify the specific subgroup of patients where they should be implemented. Moreover, bioactive compounds may be effective not only against the signaling pathways involved in the tumor process but in alleviating other associated risk factors such as obesity, intestinal dysbiosis, and/or chronic inflammation [9-13].

Saponins constitute a wide group of structurally related compounds consisting of a non-polar aglycone-triterpenoid or steroidal sapogenins-attached to hydrophilic oligosaccharide moieties. Saponins are largely distributed in the plant kingdom and are mainly found in the seeds, leaves, roots, fruits, and stems. Triterpenoid saponins have been identified in legumes (soybean, lentils, alfalfa, and chickpeas, among many others), quinoa seeds, ginseng roots, quillaja bark, or liquorice roots, whereas steroid saponins have been found in fenugreek seeds, yucca, ginseng roots, asparagus, or oats [14]. Although saponins have traditionally been recognized as anti-nutrients, 
current research is being focused on saponins and sapogenins as bioactive compounds due their biological activities, including hypocholesterolemic effects, anti-inflammatory, immunomodulatory, antibacterial, antiviral and antitumor, among others [15].

The events that take place during the gastrointestinal digestion of saponins are highly related to their bioactivities. This is because, in general, it is assumed that saponins are poorly absorbed, showing a long residence time in the intestinal tract, and hence, their potential to cause biological activities at intestinal level is increased, as activity on CRC cells might be [16]. At the colonic level, the evidences have shown that saponins are transformed by the resident microbiota into sapogenins [17]. In this sense, it is important to remark that most sapogenins have demonstrated a superior bioavailability and higher concentrations at the systemic level, and it is generally assumed that some bioactivities of sapogenins are higher than those of their former saponins [16]. As an example, diosgenin (25R-spirosten-5-en-3 $\beta$-ol) is the most studied steroidal sapogenin, fenugreek seeds being one of its richest sources, and excellent reviews have been done summarizing its biological activities [18]. Its antitumoral activities have been associated to the suppression of malignant transformation, reduction of the oxidative stress, regulation of T-cell antitumor immune response, inhibition of the epithelial-mesenchymal transition, remodeling of actin cytoskeleton, and inhibition of angiogenesis, among others [19]. As an example of a typical triterpenoid sapogenin, oleanolic acid is within the most popular ones, typically found in olive oil, but the saponins contained in quinoa seeds being recent sources of interest for this bioactive compound. The studies describing the antitumor activity of oleanolic acid have reported the inhibition of proliferation, induction of apoptosis, induction of autophagy, inhibition of infiltration, or cell cycle arrest $[20,21]$.

Due to the potential of these molecules as bioactive agents, great efforts are being made to obtain saponin and sapogenin-rich extracts using non-conventional extraction methods, such as ultrasound-assisted extraction (UAE), which reduces time and energy consumption and augments the extraction efficiency. In a recent work, we produced UAE extracts from fenugreek and quinoa in order to obtain saponin-rich extracts from these popular edible seeds rich in both types of sapogenins, namely steroid and triterpenoid saponins (FE and QE), respectively [22]. Subsequently, the acid hydrolysis of these extracts was performed in order to obtain sapogenin-rich extracts (HFE and HQE), which was proposed as an innovative technological strategy to produce novel natural extracts with potential improved bioactivity. This is because, interestingly, in addition to sapogenins, different bioactive compounds of potential interest such as phytosterols, phenolic compounds, alkylresorcinols, tocopherol, or lipids were also identified in such extracts [22].

In the present work, we aim to compare the effects of saponin-rich extracts from fenugreek and quinoa (FE and QE) and their acid hydrolysates (HFE and HQE) on the cell viability of two colorectal cancer cells (DLD1 and SW620). The effect on cell bioenergetics was analyzed by measuring mitochondrial oxidative phosphorylation and aerobic glycolysis, as well as the expression of distinct lipid metabolism targets. We demonstrate that FE and HFE diminished mitochondrial oxidative phosphorylation and aerobic glycolysis, ending up with decreased ATP levels, whereas none of the quinoa extracts (QE and HQE) showed any relevant effect at the tested doses. Importantly, we have identified distinct molecular targets implicated in the mechanism of action of the fenugreek extracts. Finally, in the frame of precision nutrition, we discuss the relevance of coexisting compounds in the extracts as permeability enhancers, and the hydrolysis transformation of the extracts as innovative strategies to augment the clinical therapeutic potential of the extracts

\section{Results}

\subsection{Saponin- and Sapogenin-Rich Extracts from Fenugreek Inhibit Cell Viability of CRC Cell Lines}

Saponins are a wide group of structurally related compounds formed by a non-polar aglycone-triterpenoid or steroidal sapogenins-attached to hydrophilic oligosaccharide moieties. For this reason, we first compared the effect of UAE extracts rich in saponins from two different 
sources-fenugreek (FE), rich in steroid saponins, and quinoa (QE), rich in triterpenoid saponins-on the inhibition of cell viability of CRC cell lines (DLD1 and SW620). Moreover, as sapogenins have been generally pointed out as superior bioactive compounds compared to their former saponins, and considering that the human gut microbiota has demonstrated to release such sapogenins [17], the acid hydrolysis of the saponin-rich extracts to obtain sapogenins-rich extracts HFE and HQE was performed to also assess their effect on cell viability. Dose-response curves and $\mathrm{IC}_{50}$ values after $48 \mathrm{~h}$ of treatment with the different extracts are shown in Figure 1. For comparison purposes, commercial extracts from fenugreek (rich in steroid saponins) and quillaja (rich in triterpenoid saponins) were included.

DLD1
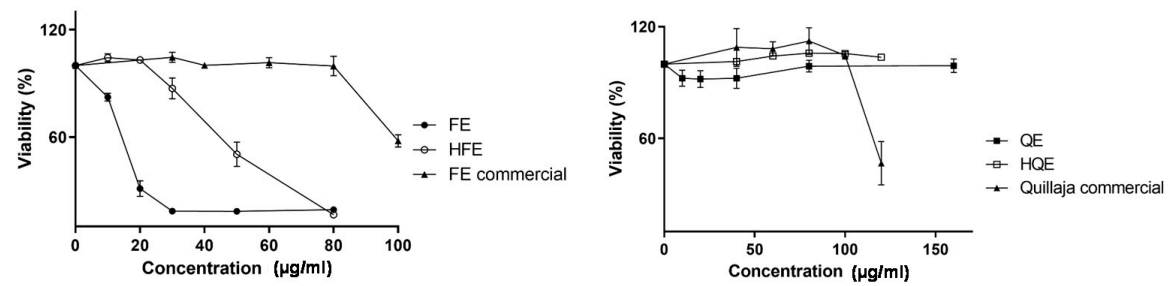

SW620
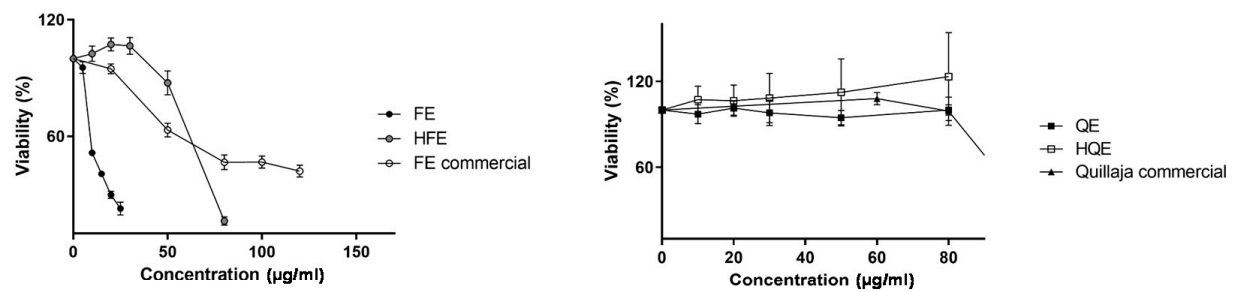

\begin{tabular}{|c|c|c|}
\hline Extract & $\begin{array}{c}\text { DLD1 } \\
\mid \mathrm{CC}_{50}(\mu \mathrm{g} / \mathrm{m} \mid)\end{array}$ & $\begin{array}{c}\text { SW620 } \\
\mid \mathrm{IC}_{50}(\mu \mathrm{g} / \mathrm{ml})\end{array}$ \\
\hline FE (steroid saponins) & $19.2 \pm 1.2$ & $12.8 \pm 1.4$ \\
\hline HFE (sapogenin rich) & $51.6 \pm 3.4$ & $60.3 \pm 1.8$ \\
\hline QE (triterpenoid saponins) & $>100$ & $>100$ \\
\hline HQE (sapogenin rich) & $>100$ & $97.5 \pm 2.6$ \\
\hline Qui (commercial) & $>100$ & $91.5 \pm 4.7$ \\
\hline FG (commercial) & $>100$ & $>100$ \\
\hline
\end{tabular}

Figure 1. Dose-response curves and $\mathrm{IC}_{50}$ values of different saponin- and sapogenin- rich extracts on colorectal cancer (CRC) cell lines pretreated for $48 \mathrm{~h}$ with fenugreek and (FE), hydrolyzed fenugreek extract (HFE), quinoa (QE) and hydrolyzed quinoa extract (HQE) compared to control non-treated cells. For comparison purposes, two commercial extracts rich in steroid saponins (Fenfuro) and triterpenoid saponins (Quillaja) are shown.

QE showed no effects on the inhibition of the cell viability of CRC cell lines in the range of concentration assayed $(0-120 \mu \mathrm{g} / \mathrm{mL})$. HQE showed IC $_{50}$ values close to $100 \mu \mathrm{g} / \mathrm{mL}$. In line with these results, Qui, with saponins of the same triterpenoid nature, presented $\mathrm{IC}_{50}$ values in the similar range of HQE.

In contrast, $\mathrm{FE}$, rich in steroid saponins, showed $\mathrm{IC}_{50}$ values below $20 \mu \mathrm{g} / \mathrm{mL}$ in both cell lines. HFE also showed significant effects on cell viability, although the $\mathrm{IC}_{50}$ values were 2.5 times higher than those of the non-hydrolyzed extract.

Finally, the commercial fenugreek extract was less effective than the experimental $\mathrm{FE}$, showing $\mathrm{IC}_{50}$ values $>100 \mu \mathrm{g} / \mathrm{mL}$. This extract contained $50 \%$ saponins, according to specifications. Unfortunately, neither a detailed and complete characterization of this extract in other compounds nor the procedure of extraction and fractionation of this commercial extract were available. 


\subsection{Saponin- and Sapogenin-Rich Extracts from Fenugreek Diminish Cell Bioenergetics of CRC Cell Lines}

In recent years, there is increasing interest on targeting the altered cancer metabolism. For this reason, and taking into account the interesting results observed in cell viability for the experimental fenugreek extracts, we aimed to investigate if the UAE saponin-rich extract FE and its hydrolysate HFE could target cancer cell metabolism, and more specifically the energetic metabolism (glycolysis and mitochondrial respiration) of colorectal cancer cells.

\subsubsection{FE and HFE Diminished the Mitochondrial Oxidative Phosphorylation of CRC Cell Lines}

First, we investigated the impact of saponin-rich extracts on the mitochondrial oxidative phosphorylation of colorectal cancer cells (DLD1 and SW620). By means of the Extracellular Flux Bioanalyzer (Seahorse Bioscience), we monitored the oxygen consumption rates (OCR) after the sequential addition of different drugs that regulate the mitochondrial function.

The study of cell bioenergetics (mitochondrial oxidative phosphorylation and aerobic glycolysis) was performed after treatment CRC cells for $48 \mathrm{~h}$ with different doses of FE and HFE extracts corresponding to $1 / 2 \times \mathrm{IC}_{50}\left(1 / 4 \times \mathrm{LC}_{50}\right)$ and $1 \times \mathrm{IC}_{50}\left(1 / 2 \times \mathrm{LC}_{50}\right)$. Non-treated cells were kept as controls. Importantly, before running the experiments, the same number of non-treated cells and pre-treated cells were plated in a XFe Seahorse plate in complete media (DMEM, 10\% FBS) for $6 \mathrm{~h}$ to allow the cells to attach, without any treatment, in order to compare the cell bioenergetics only of viable cells (DLD1: 25,000 cells/well, SW620: 40,000 cells/well). Then, the medium of the cells was changed to the non-buffered XFe Base media supplemented with $10 \mathrm{mM}$ glucose, $2 \mathrm{mM}$ glutamine, and $1 \mathrm{mM}$ pyruvate, and cells were incubated for $1 \mathrm{~h}$ at $37^{\circ} \mathrm{C}$ without $\mathrm{CO}_{2}$.

As it can be observed in Figure 2A (bioenergetic profile), DLD1 CRC cells pre-treated with FE and HFE showed reduced basal respiration rates (BRR) compared to control non-treated cells (measurements 1 to 3 ) at all the doses tested. After an injection of oligomycin, in order to estimate the OCR dedicated to ATP production, FE and HFE pre-treated cells displayed reduced levels of ATP compared to control non-treated cells (measurements 4 to 6). The maximal respiration rate (MRR) (measurements 7 to 9), after the injection of FCCP, was also affected in FE and HFE pre-treated cells. Finally, rotenone and antimycin A, inhibitors of complex I and III of the electron transport chain (ETC), respectively, were injected to shut down the OCR due to mitochondrial oxidative phosphorylation (measurements 10 to 12). These results indicate that FE and HFE clearly compromise mitochondrial respiration. Similar results were obtained in SW620 CRC cells (Figure 2B).

These effects were not observed in DLD1 CRC pre-treated cells with QE in accordance to the lack of effects on cell viability at the doses tested (Figure S1).

\subsubsection{FE and HFE Inhibit Aerobic Glycolysis of CRC}

To analyze the effect of fenugreek extracts on aerobic glycolysis, we monitored the Extracellular Acidification Rate (ECAR), which is an indirect readout of the L-lactate production, after the sequential injection of modulators of the aerobic glycolysis. DLD1 or SW620 cells were pre-treated for $48 \mathrm{~h}$ with two different doses of FE or HFE corresponding to $1 / 2 \times \mathrm{IC}_{50}$ and $1 \times \mathrm{IC}_{50}$. Non-treated cells were kept as controls.

As it can be observed in Figure 3A (upper panel), basal ECARs of FE and HFE DLD1 pre-treated cells (1 to 3 measurements) were diminished compared to control non-treated cells. Next, we injected glucose (10 mM final concentration) to monitor cells' ability to upregulate glycolysis when glucose is available. After the glucose injection, only HFE pre-treated cells had diminished levels of ECAR compared to control cells (3 to 6 measurements), indicating a reduced capacity for glycolysis. Then, oligomycin was injected to block the ATP production from mitochondria and so, to determine the Maximal Glycolytic Capacity. Pre-treated cells with FE and HFE at the higher doses $\left(1 \times \mathrm{IC}_{50}\right)$ diminished maximal ECAR levels compared to the control cells (measurements 6 to 9). 
A

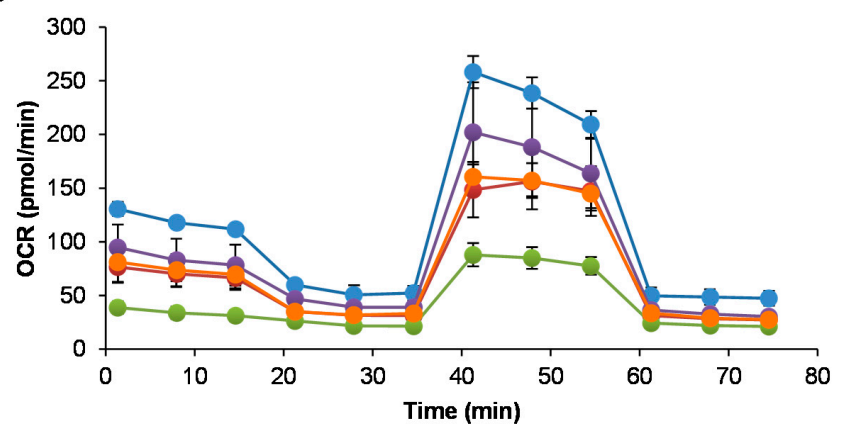

a Control

- FE- $1 / 2 \times 1 C_{50}$

FE- $1 \times \mid C_{50}$

- HFE-1/2XIC $\mathrm{C}_{50}$

HFE-1X|C
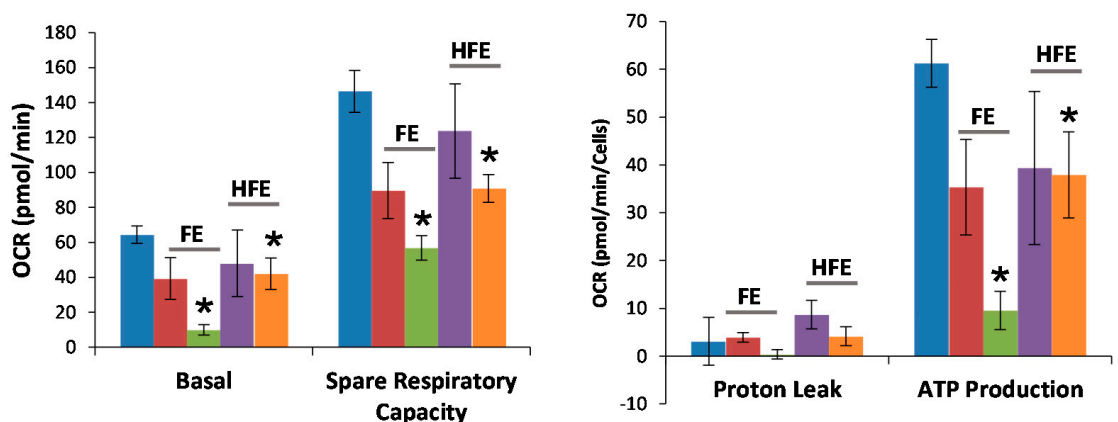

B
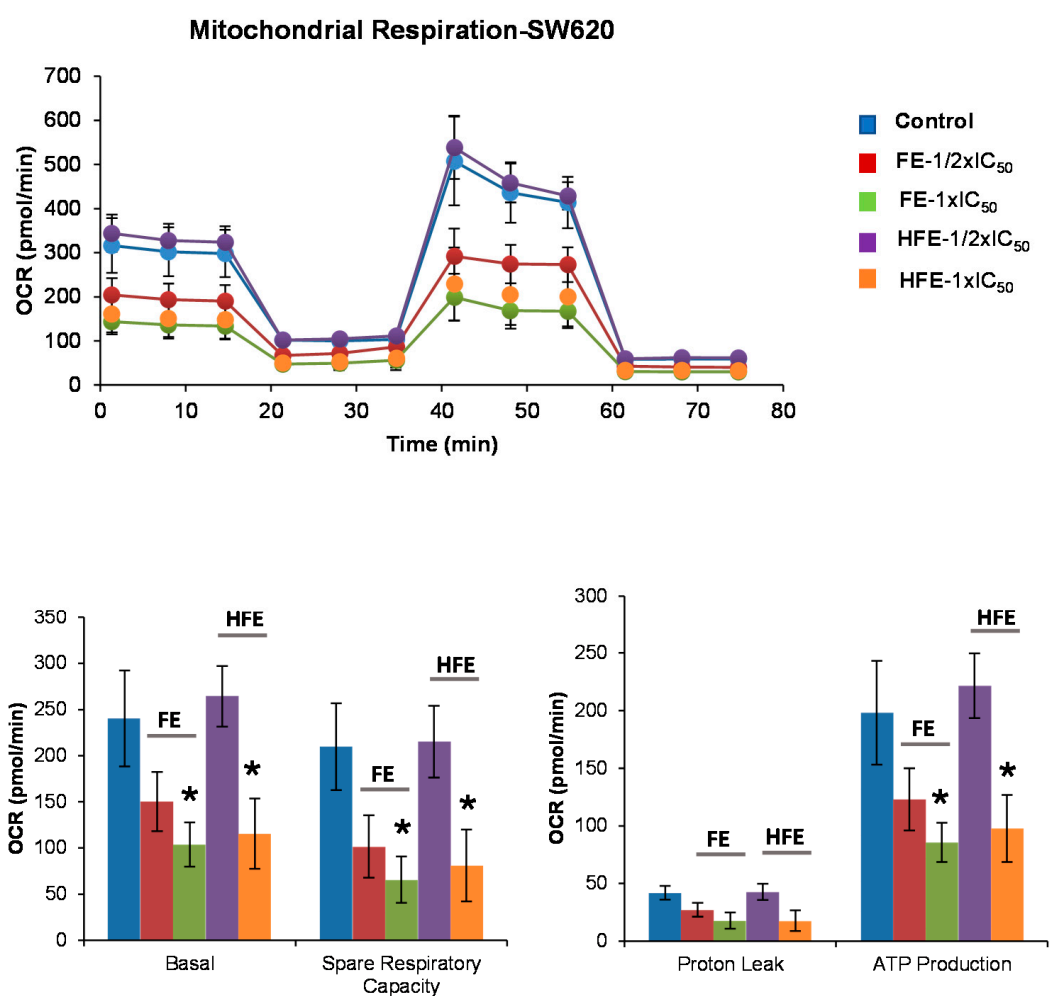

Figure 2. FE and HFE extracts inhibit mitochondrial oxidative phosphorylation of CRC cell lines. (A) MitoStress profile and quantification of basal oxygen consumption rates (OCR), Maximal Respiration Rate, ATP, Spare respiratory capacity, and Proton Leak of DLD1 CRC cells pre-treated with FE and HFE (at the indicated doses); (B) MitoStress profile and quantification of basal OCR, Maximal Respiration Rate, ATP production, Spare respiratory capacity, and Proton Leak of SW620 CRC cells pre-treated with FE and HFE (at the indicated doses). Asterisks indicate statistically significant differences $\left(p<0.05\left(^{*}\right)\right)$ relative to the control non-treated cells (6-8 replicates per condition). 
A
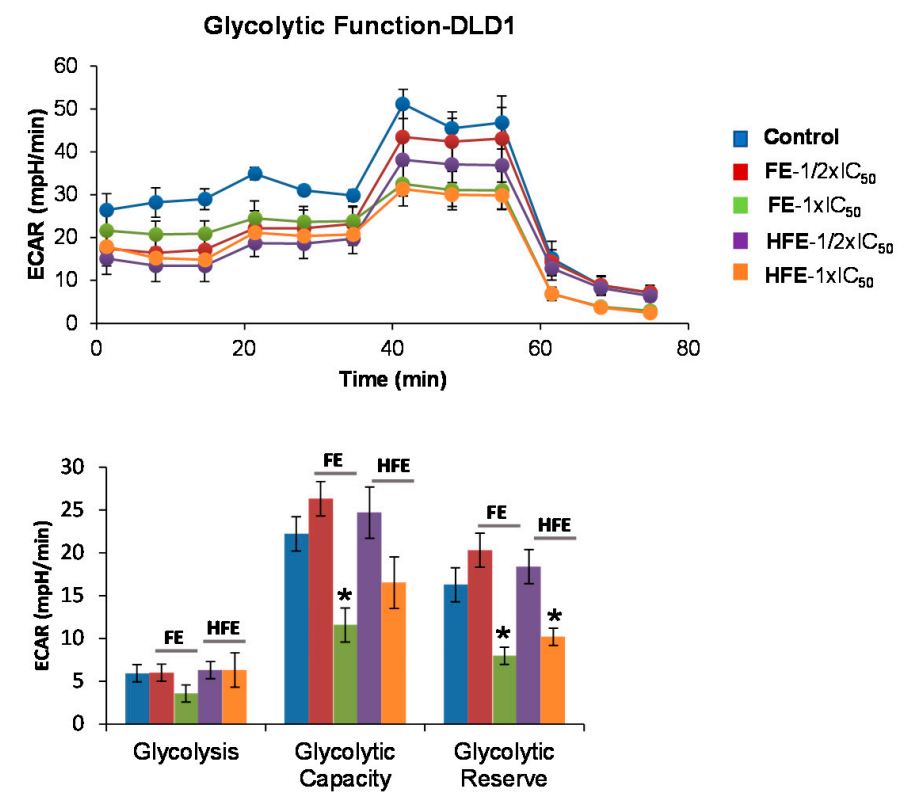

B

Glycolytic Function-SW620
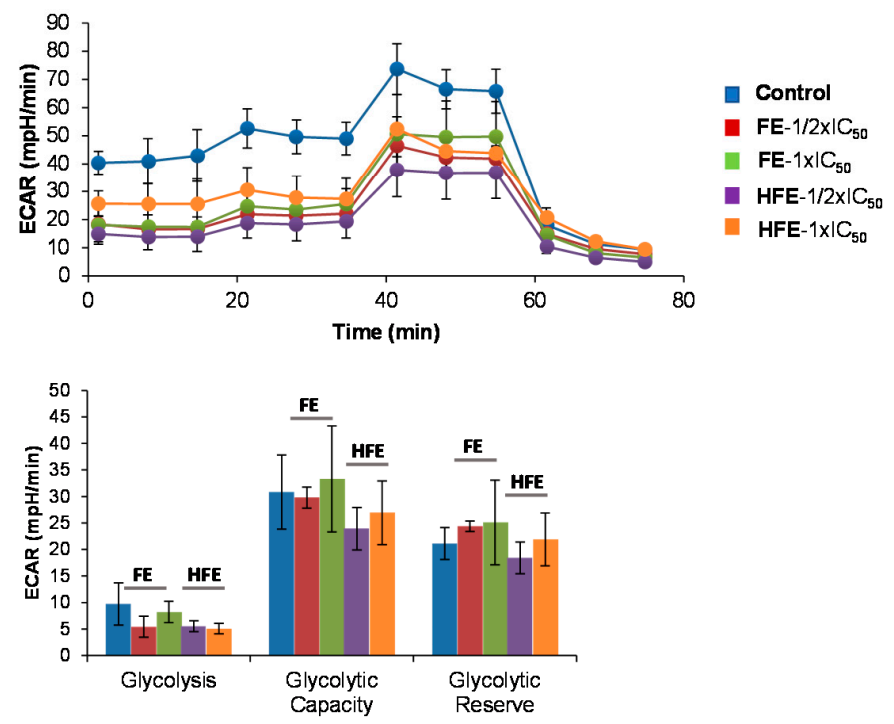

Figure 3. FE and HFE extracts inhibit aerobic glycolysis of CRC cell lines. (A) GlycoStress profile and quantification of basal ECAR, Maximal ECAR, and Glycolytic Reserve of DLD1 CRC cells pre-treated with FE and HFE (at the indicated doses). (B) GlycoStress profile and quantification of basal ECAR, Maximal ECAR, and Glycolytic Reserve of SW620 CRC cells pre-treated with FE and HFE (at the indicated doses). Asterisks indicate statistically significant differences $\left.\left(p<0.05{ }^{*}\right)\right)$ relative to the control non-treated cells (6-8 replicates per condition).

Finally, 2-DG was injected (50 $\mathrm{mM}$ final concentration) to shut down aerobic glycolysis and to determine the non-glycolytic ECAR.

Treatment SW620 cells with FE and HFE had little effect on inhibiting aerobic glycolysis (Figure 3B). Basal ECAR (measurements 1-3), quantified as the measurement 3, was not significantly diminished by the treatments. Nor the aerobic glycolysis (difference between basal ECAR and upregulated ECAR after glucose injection-measurements 4-6), nor the stressed ECAR (maximal ECAR after oligomycin injection-measurements 7-9). 
FE and HFE had limited effect on targeting aerobic glycolysis of SW620 CRC cell lines (Figure 3B), reflecting different metabolic performance between DLD1 and SW620 CRC cell lines.

As HIF $1 \alpha$ is a key transcription factor promoting aerobic glycolysis, we also wanted to evaluate the effects of FE and HFE in the regulation of the expression of HIF1A and LDHA. No significant effects were found after treatment with FE or HFE, except for $L D H A$, which was significantly downregulated by FE at $30 \mu \mathrm{g} / \mathrm{mL}(p=0.0264)$.

In summary, the analysis of cell bioenergetic profile of CRC cell lines pre-treated with FE and HFE extracts indicated that both extracts had a direct effect on diminishing bioenergetic pathways in CRC, with a higher impact on the inhibition of mitochondrial oxidative phosphorylation.

Quantification of the intracellular ATP levels confirmed the effects of FE and HFE in the inhibition of CRC cell bioenergetics (Figure S2).

\subsection{FE and HFE Inhibit CRC Metabolism by Targeting Distinct Molecular Pathways}

The role of lipid metabolism alterations in cancer has been recently highlighted. In this regard, SREBP proteins, which are master regulators of lipid metabolism, together with FASN and SCD have been found to be upregulated in several neoplasias. Previous work by the group has identified the association between lipid metabolism genes and the prognosis of colon cancer patients, which provides a way to personalize the treatment of these patients [23,24].

In addition, current evidence shows that up to one-third of cancer deaths could be prevented by modifying risk factors, such as the consumption of saturated fatty acids. Moreover, the risk of death by cancer is increased 1.5-1.6 in individuals with a body mass index (BMI) $>40 \mathrm{~kg} / \mathrm{m}^{2}$ [25]. Obesity induces a low grade of chronic inflammation promoting the carcinogenic process, as well as diminishing the response to antitumor treatments $[26,27]$.

With the objective of evaluating the effect of FE and HFE on CRC cancer metabolism and risk factors related to obesity and inflammation, we designed a panel of metabolic genes-including de novo lipogenesis and cholesterogenesis (SREBF1, FASN; SCD; SREBF2, HMGCR), fatty acid metabolism (ACSL1, ACSL4, SCD), oncogenic pathways in CRC (CHOKA, BMP2), exogenous uptake of lipids (LDLR, FABP1), cholesterol metabolism (ABCA1, ApoA1), inflammation and oxidative stress (JAK1, $N E F 2 L 2)$, and resistance to chemotherapy (TK1, TYMS). In order to validate the specificity of the effect of the different extracts, we included in the analysis a supercritical rosemary extract (RE), whose molecular effects on these metabolic targets have been previously analyzed [7].

Interestingly, FE and HFE differently modulated the expression of lipid metabolism genes in DLD1 CRC cells pre-treated for $48 \mathrm{~h}$ with the extracts (Figure 4).

FE diminished the expression of $A B C A 1$ implicated in the reverse transporter of cholesterol, which has been associated with poor prognosis in CRC patients [4]. Interestingly, FE augmented the expression of genes associated to the exogenous uptake of fatty acids and low-density lipoproteins (LDL), as well as genes related to de novo lipogenesis and cholesterogenesis. These results suggest that FE may promote anabolism, which may be of interest in cancer patients developing cachexia. In addition, FE significantly downregulated genes related to the synthesis of nucleic acids-TYMS and TK1, which have been implicated in the appearance of resistance to the anti-metabolite 5-fluorouracil [28]. In order to determine if FE may synergize with the chemotherapeutic drug 5-fluorouracil (5-FU), we pre-treated CRC cells for $4 \mathrm{~h}$ with FE ( $5 \mu \mathrm{g} / \mathrm{mL}$ corresponding to $1 / 4$ of the $\mathrm{IC}_{50}$ ) before the addition of 5-FU at different doses: 5,10, and $15 \mu \mathrm{g} / \mathrm{mL}$. The combination index indicated a positive synergism between FE and 5-FU (Figure S3).

On the other hand, HFE diminished lipid metabolism targets implicated in de novo lipogenesis and cholesterogenesis (including SREBF1, FASN, SCD, SREBF2, and HMGCR), and two metabolic axes-ABCA1 [4] and ACSL1/ACSL4/SCD network [3]-were described to promote invasion and migration and to correlate with poorer prognosis in CRC patients. Moreover, $F A B P 1$-free fatty acid binding protein—and $B M P 2$ were also downregulated. 

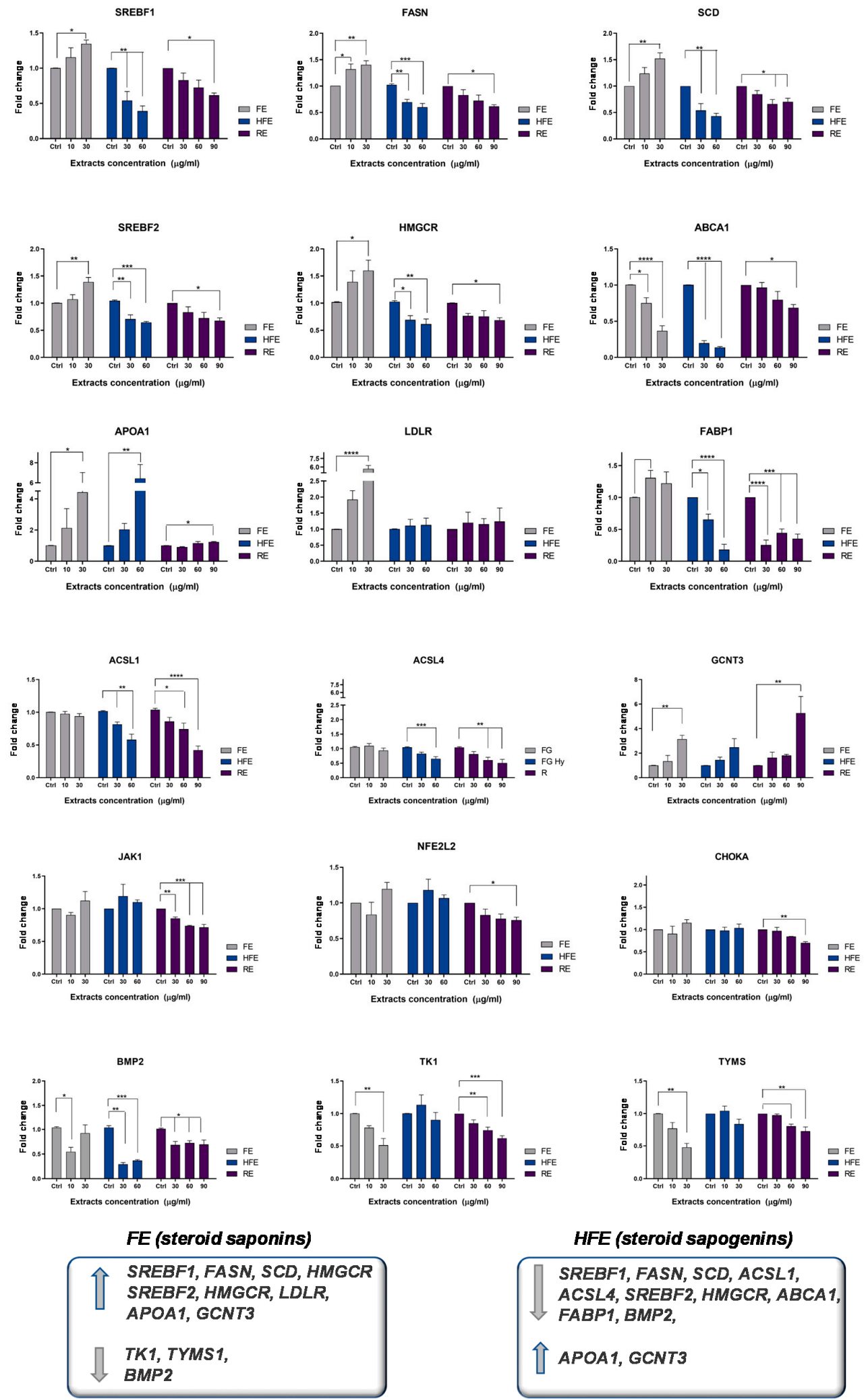

Figure 4. FE and HFE extracts differentially regulate metabolic targets in the DLD1 CRC cell line. For specificity of the molecular targets, the effects of a well-characterized supercritical rosemary extract (RE) is shown. Asterisks indicate statistically significant differences $\left(p<0.05\left(^{*}\right) ; p<0.01\left(^{* *}\right)\right.$; $p<0.005\left(^{* * *}\right) ; p<0.001\left(^{* * *}\right)$ ) relative to the control non-treated cells ( $3-4$ replicates, three independent experiments). 
In line with these results, DLD1 CRC cells treated with HFE for $48 \mathrm{~h}$ were less prone to accumulate neutral lipid droplets compared to control non-treated cells (DMSO) (Figure S4).

Both extracts augmented the expression of GCNT3, which has been described as a good prognosis biomarker in CRC [29], and ApoA1, described to counteract the protumoral effects of ABCA1 overexpression [4].

These results were validated in a second CRC cell line SW620 (Figure 5).
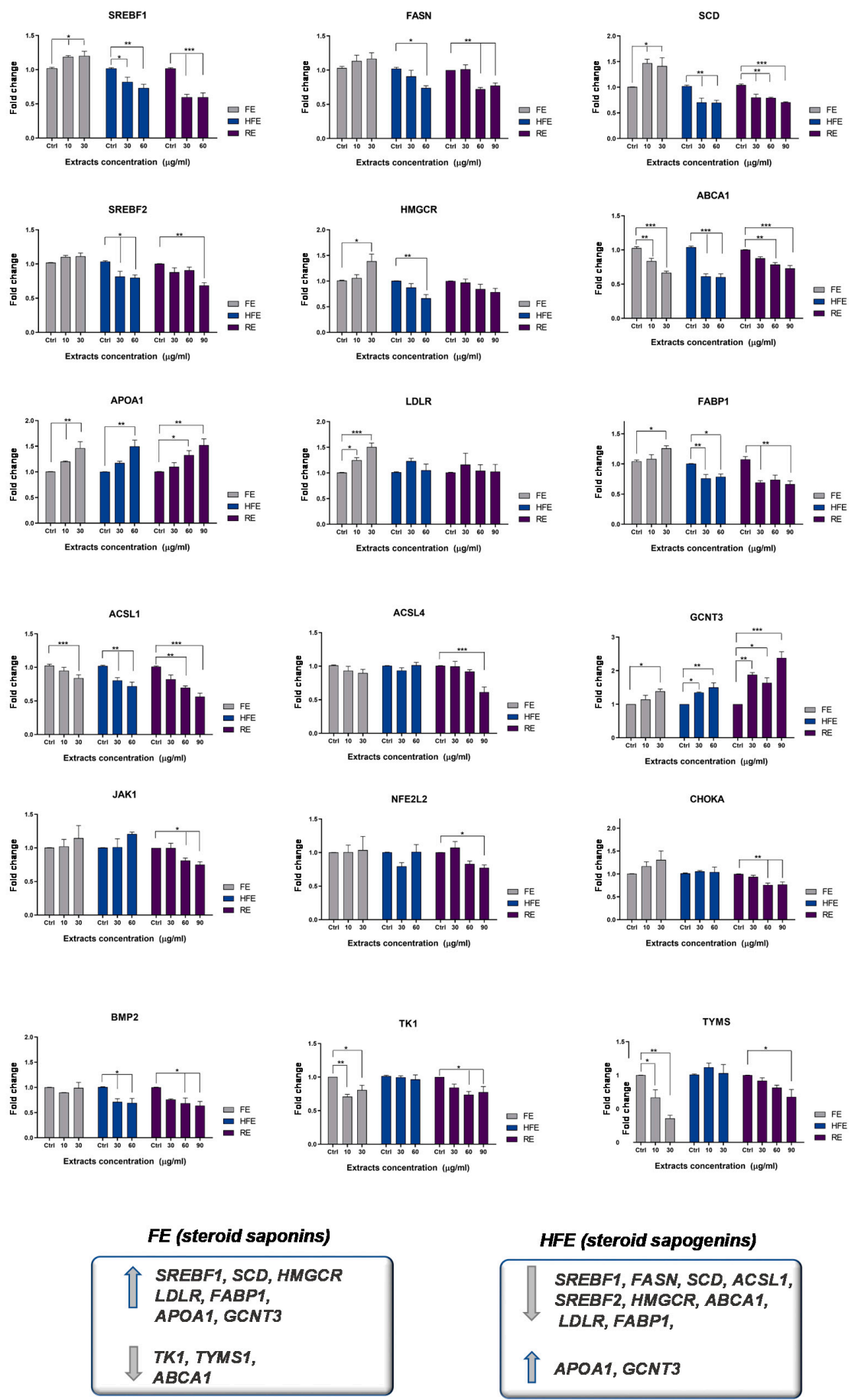

Figure 5. FE and HFE extracts differentially regulate metabolic targets in SW620 CRC cell line. For specificity of the molecular targets, the effects of a well-characterized supercritical rosemary extract (RE) is shown. Asterisks indicate statistically significant differences $\left(p<0.05\left(^{*}\right) ; p<0.01\left({ }^{*}\right) ; p<0.005\right.$ $(* * *))$ relative to the control non-treated cells (3-4 replicates, three independent experiments). 


\section{Discussion}

The increase in the incidence of chronic diseases associated to metabolism such as obesity, insulin resistance, cardiovascular diseases, and cancer is a social and medical matter of concern. Related to cancer, evidence from epidemiological and clinical trials have shown that up to one-third of cancer deaths could be prevented by modifying key risk factors, diet and exercise being among the most important factors, due to their association with obesity $[30,31]$. The altered systemic metabolic function during obesity, including low-grade chronic inflammation, insulin resistance, hypercholesterolemia, and hypertriglyceridemia, seem to be on the basis of the association between obesity and the carcinogenic process.

The reprogramming of metabolism is a key event in tumorigenesis [1], and efforts are being oriented to identify molecular targets and oncogenic pathways sustaining the altered metabolism in cancer. Together with the promotion of aerobic glycolysis (Warburg effect) and the increased glutaminolysis, the relevance of lipid metabolism in cancer has been extensively demonstrated $[3,32,33]$. Lipids, in addition to their role as fuels for ATP production, play a key role as components of plasmatic and organelles membranes, affecting their plasticity, and they are also important as cell signaling molecules. Lipid metabolism alterations have been associated with tumor progression and malignancy. In this regard, SREBP proteins, which are master regulators of lipid metabolism, together with FASN and SCD have been found to be upregulated in several neoplasias [32].

According to GLOBOCAN 2018 data, CRC is the third most deadly and fourth most commonly diagnosed cancer in the world [34]. In the course of this multifactorial condition, numerous alterations occur both in tumor suppressor and oncogenic genes. Experimental and epidemiological studies support the role of lipid metabolism, diet, gene-diet interactions, obesity, and gut microbiota in the etiology and prognosis in CRC [35].

Key enzymes of lipid metabolism are differentially expressed in normal and tumor CRC tissues. Some of them are associated with cancer survival and have been individually proposed as prognosis markers [35]. Lipid metabolism alterations not only affect the primary tumor but also influence malignancy, migration, invasion, relapse, and resistance to chemotherapy treatment. A lipid-metabolic signature ColoLipidGene is associated with CRC prognosis in stage II patients [23]. A lipid metabolic network formed by ACSL1, ACSL4 (Acyl-CoA synthetases), and SCD promotes migration and invasion by CRC cells [3]. Epidemiological studies have linked cholesterol levels with the risk of developing different tumors [36-38]. The formation of high-density lipoproteins (HDL) for reverse cholesterol transport is based on the action of the ABCA1 transporter, which has been recently associated with poor prognosis in colon cancer patients [4]. In addition, genetic analysis of 57 SNPs located in seven lipid metabolism locations has shown that rs8086 SNP in ACSL1 was associated with reduced mRNA expression levels of ACSL1 and shorter CRC disease-free survival (DFS) [39]. In addition, evidence has demonstrated that nearly half of the cancer deaths could be prevented by modifying key risk factors related to lifestyle (smoking, alcohol consumption, high saturated fatty acid intake) [40,41].

Efforts are being oriented to develop therapeutic strategies against cancer metabolism. Vegetables and plants are among the most popular sources to obtain bioactive compounds. Phytochemicals exert numerous biological activities, such as anti-inflammatory, antihypertensive, antioxidant, anticarcinogenic, antidiabetic, or antiobesity [42]. Thus, targeted nutritional interventions based on the knowledge of the molecular mechanism of action and molecular targets of bioactive compounds and/or ingredients from diet may contribute to enhance cancer therapy and the survival of cancer patients.

In this sense, we recently produced extracts from the edible seed fenugreek (FE, HFE) and quinoa (QE, HQE) with a relevant content of different bioactive compounds, including saponins and sapogenins within the major bioactive components of the non-hydrolyzed and hydrolyzed extracts, respectively. Additionally, these extracts also contained other interesting compounds whose contribution to the final bioactivity might be of interest [22]. In general, saponin-rich extracts from both seeds also contained amino acids, glycerides, and carbohydrates, as well as phytosterols, tocols, and phenolic compounds. 
On the contrary, both sapogenin-rich extracts mainly contained free fatty acids and partial glycerides, and they were richer in phytosterols, phenolics, and tocols, and they did not contain any amino acids or carbohydrates. All four extracts have also shown promising effects on lipid absorption by inhibiting the pancreatic lipase, whereas both hydrolyzed extracts have very recently demonstrated to interfere with the bioaccessibility of cholesterol [43]. Even FE has shown a potential prebiotic-like effect [17]. Hence, the demonstrated multibioactivity of these extracts at different levels of the gastrointestinal tract motivated the further evaluation of these extracts as potential anticancer agents in colorectal cancer as an additional potential bioactivity at the gastrointestinal level. The suggested long residence time of saponins at this tract as well as the demonstrated biotransformation of saponins to sapogenins by microbiota were motivators as well [17].

As shown in Figure 1, QE showed no effects on the inhibition of the cell viability of CRC cell lines in the range of concentration assayed $(0-120 \mu \mathrm{g} / \mathrm{mL})$. HQE showed $\mathrm{IC}_{50}$ values close to $100 \mu \mathrm{g} / \mathrm{mL}$. In line with these results, commercial quillaja (Qui), containing saponins of the same nature as those in quinoa, presented $\mathrm{IC}_{50}$ values in the similar range of $\mathrm{HQE}$ extract. These results suggest that $\mathrm{QE}$ and HQE extracts have little impact on the inhibition of proliferation of CRC cells (DLD1 and SW620).

In contrast, $\mathrm{FE}$, rich in steroid saponins, showed $\mathrm{IC}_{50}$ values below $20 \mu \mathrm{g} / \mathrm{mL}$. HFE also showed significant effects on cell viability, although the $\mathrm{IC}_{50}$ values were 2.5 times higher than those of the non-hydrolyzed extract.

Despite the fact that the HFE extract was the one that contained free sapogenins, which are considered as the most bioactive derivatives, the higher effect observed with FE suggests the presence of additional compounds not present in the HFE, acting synergistically with the steroid saponins on the inhibition of CRC cell viability. Additionally, the concentration of active compounds in both extracts should be also considered. The previous detailed characterization of FE and HFE showed important differences both in the phytochemical composition and in the concentration of saponins and sapogenins [22]. Thus, whereas saponins were one of the major components quantified in FE $(31 \%)$, the sapogenin content of HFE was much lower $(8 \%)$. FE also contained amino acids and carbohydrates, which were not present in case of HFE because, in general, HFE was mainly a less polar extract that was especially rich in free fatty acids (35\%) such as linoleic and oleic acids and partial glycerides [22]. In this sense, it is interesting to remark the recent study performed by Storniolo et al. [44] where oleic acid promoted mitogenic activity in colon cancer cell lines, whereas olive oil, rich in this fatty acid, demonstrated protective effects against this disease. These contradictory results have been attributed to the protective effects of additional minor bioactive components present in olive oil, which seem to counteract the mitogenic effects of oleic acid alone. Similarly, it can be hypothesized that the minor bioactive components of HFE, such as sapogenins alone and/or in synergism with co-existing compounds, might be actively exerting antiproliferative effects regardless of the high free fatty acid content. In agreement, the exceedingly lower content of linoleic and oleic acids in FE (1.44\%) might help partially explaining the superior bioactivity of this extract respect to HFE. Further studies in this respect might be worth exploring in order to elucidate the effects of co-extracted components on the antiproliferative activity of these extracts.

By means of the latest technology in the analysis of cell bioenergetics, we demonstrated that FE and HFE target CRC cell bioenergetics by diminishing mitochondrial oxidative phosphorylation (Figure 2A,B) and aerobic glycolysis (Figure 3A,B), ending up with a decrease in the levels of ATP (Figure S2). As far as our knowledge goes, no previous studies have been performed in which the cell bioenergetics have been assessed with extracts where saponins and sapogenins are within the major bioactive components or with fenugreek extracts in general. Only a couple studies have very recently the steroid saponin gracillin as a mitochondria-targeting antitumor drug, thanks to its ability to induce a dose-dependent decrease of mitochondrial respiration and the inhibition of aerobic glycolysis $[45,46]$.

Importantly, we have identified in this study distinct molecular targets implicated in the mechanism of action of the extracts. FE inhibited the expression of TYMS and TK1, which have been associated to the appearance to resistance to 5-FU. FE also synergized with 5-FU on the inhibition of CRC cell viability 
(Figure S3). Interestingly, we also found that FE upregulated lipid metabolism targets, such as SREBF1, FASN, SCD SREBF2, HMGCR, and LDLR, which suggest an anabolic effect for this extract. Very recently, Mohammad-Sadeghipour, Mahmoodi, Karimabad, and Mirzaei [47] found that a hydroalcoholic extract obtained from fenugreek also upregulated the expression of $L D L R$ in SW480 colorectal cancer cell lines, although the expression of FASN was downregulated by the extract, as opposed to our findings. However, Zhout et al. [48] did find similar outcomes in healthy tissues. Specifically, an alfalfa saponin extract was able to upregulate the expression of FASN in hepatic tissue from laying hens. The upregulation of $L D L R$ by an alfalfa saponin extract has also been demonstrated in healthy buffalo rat liver cells [49]. These results highlight the relevance of additional bioactive compounds of saponin-rich extracts contributing to define the specific targeted lipid metabolism network.

In addition, FE upregulated the expression of GCNT3, which has been proposed as a biomarker of good prognosis [29,50], and ApoA1, which has shown to counteract the effects of ABCA1 in the promotion of invasiveness and stemness [4]. No previous studies have been found regarding the upregulation of GCNT3 by fenugreek or saponins, nor the upregulation of APOA1.

On the other hand, HFE inhibited lipid metabolism targets, including SREBF1, FASN, SCD, SREBF2, HMGCR, LDLR, FABP1, ABCA1, and the metabolic axis ACSL/SCD, all of which have been implicated in poorer prognosis in CRC [3]. In line with these results, CRC cells treated with HFE showed a reduced lipid content compared to control non-treated cells (Figure S4). Mohammad-Sadeghipour, Mahmoodi, Karimabad, and Mirzaei [47] have also described the downregulation of the expression of FASN FAS by diosgenin, which appears to be the major aglycone in the HFE, although the expression of $L D L R$ was upregulated by this sapogenin, as opposed to our findings. These results indicate that additional bioactive compounds are contributing to define the specific lipid targets modulated by whole extracts.

It is important to remark that bioactive compounds exert their cytotoxic activity at specific regions of the gastrointestinal tract, depending on the bioaccessibility and cellular uptake at the intestinal level. In this sense, low bioaccessibility and cellular permeabilities would be required at the small intestine level if the targeted cancerous tissues are located in the last parts of the intestinal tract, such as colon. In general, a low intestinal permeability and poor bioavailability has been described for saponins, suggesting that these compounds would mainly reach the colon intact to exert their cytotoxic effect locally. In the case of sapogenins, they are also considered to have low bioavailability due to low aqueous solubility and poor bioaccessibility. However, these general ideas should not be widely assumed, since contrary outcomes have been observed in this regard. Thus, in the specific case of the experimental fenugreek extracts assayed, we previously demonstrated that saponins from FE had a complete bioaccessibility [22] and high in vitro membrane permeability [51] after the simulation of gastrointestinal digestion of this extract, which suggested that saponins from these extracts would be likely absorbed at first sections of the intestinal tract. This result was explained by the coexistence of other compounds in the extracts that might be acting as "permeability enhancers". Specifically, coexisting lipids in the extracts were demonstrated as one of the permeability enhancers of saponins of FE [51], which provide new possibilities for the delivery of these bioactive compounds at different parts of the intestinal tract: (i) if it is required for saponins to act at the systemic level, the presence of co-existing permeability enhancers should be sought to augment permeability at the small intestine, as it was the case of FE; (ii) if it is required for saponins to target cancerous tissues located in the last parts of the intestinal tract, such as the colon, a low intestinal permeability and poor bioavailability should be sought. Considering these approaches, our results suggest that in the case of CRC patients developing cachexia, the high bioaccessibility and permeability of saponins from FE at the intestinal level (small intestine) would allow this extract to be a good candidate to promote anabolic effects systemically, taking into account the relevant results observed for SREBF1, $F A S N, S C D$, and LDLR, among others, for this extract. On the other hand, in the case of CRC patients where lipid metabolism-related genes associated to progression and metastasis such as $S R E B F 1$, FASN, $A B C A 1$, and the ACSL/SCD axis, among others, are overexpressed, it should be desirable to 
develop formulas to deliver FE to the colon in order to be metabolized by the colonic microbiota to release the aglycone sapogenins. In this sense, recent studies have confirmed that the human gut microbiota is capable of releasing the aglycones from saponins contained in FE [17], which is a situation that might allow sapogenins to act locally on colon cancer cells. However, alternatively to the development of colon-delivery formula, the production of HFE would be also an interesting strategy to act on such lipid metabolism genes at the colon level. This is because our previous studies showed that despite sapogenins from HFE having a good bioaccessibility [22], these compounds showed a poor in vitro membrane permeability [51]. Therefore, it might be expected that sapogenins from HFE would reach the last sections of the intestinal tract, allowing to exert their local effect on lipid metabolism-related genes in CRC cells. Nevertheless, further studies would be necessary in order to evaluate whether additional strategies are necessary to favor their permeability at the specific site of colon cells. According to our previous study, co-existing lipids would also be proper permeability enhancers for fenugreek sapogenins.

Therefore, the distinct metabolic mechanisms of FE and HFE in targeting CRC metabolism suggest distinct scenarios in combination with specific technological strategies, such as the hydrolysis of extracts or formulations, in order to deliver the bioactive compounds at specific regions of the gastrointestinal tract to exert their bioactivity.

Finally, in the frame of precision nutrition, additional aspects have to be taken into consideration, as they will refine the final responses to specific nutritional intervention, such as the composition of the intestinal microbiome, the nutritional status of individuals, and/or the metabolic alterations at the systemic levels (obesity, T2DM, CDV, cancer ... ). Bioactive compounds and ingredients from the diet have a direct effect on the composition and diversity of the intestinal microbiota, as demonstrated for the assayed FE [51]. Importantly, numerous metabolites derived from the microbiota have been shown to regulate local inflammation as well as the innate and adaptive immune responses. In these sense, additional challenges are oriented to understand how diet might impact cancer development through modulating the composition of gut microbiota, the metabolites derived from microbiota, and the intestinal immune system functionality. Therefore, further studies will be oriented to explore whether the sapogenins released from FE by the microbiota, which has been demonstrated previously, and the modulation of the composition of microbiota by FE [51] would impact local inflammation and immune responses. The equilibrium between inflammation and tolerance in the gut has been shown to modulate precancerous lesions to progress or not into cancer [52]. In a similar way, the nutritional status of individuals (obesity, dyslipidemias) also impacts on the differential responses of individuals by triggering low-grade chronic inflammation and affecting the systemic energy balance. Numerous studies describe the role of chronic inflammation and the immune system in the carcinogenic process, as well as in the response to antitumor treatments. In this sense, the possibility of modulating the innate immune response, which stems at the intestinal level, through nutrition is receiving great interest in the treatment of cancer. Proper manipulation of this type of response could enhance other antitumor therapies and immunotherapies [53].

\section{Materials and Methods}

\subsection{Saponin-Rich Extracts and Sapogenin-Rich Extracts}

Seeds of red quinoa (Chenopodium quinoa) were purchased from Hijo de Macario Marcos (Salamanca, Spain) and seeds of fenugreek (Trigonella foenum-graecum) were purchased from Murciana de Herboristeria (Murcia, Spain).

The preparation of saponin-rich extracts was previously performed by Navarro, Reglero \& Martin [22] by UAE with methanol and subsequent concentration of saponins with water and 1-butanol. The final dried extracts showed a saponin content of $31 \%$ for $\mathrm{FE}$ and $7.9 \%$ for $\mathrm{QE}$, according to the HPLC-DAD analysis [22]. Subsequently, sapogenin-rich extracts were also obtained in the same previous study by acid hydrolysis and followed by an extraction of sapogenins with ethyl acetate. 
The final dried extracts showed a sapogenin content of $8 \%$ for HFE and $5.4 \%$ for HQE, according to the GC-MS analysis [22].

For comparative purposes, quillaja extract ( $20 \% w / w)$ (Qui) from Sigma Aldrich and commercial fenugreek extract from Biotanica ( $50 \%$ w/w, Aucklan, New Zealand) were used as reference commercial products of triterpenoid and steroid saponin-rich extracts, respectively. Supercritical-rosemary extract (RE) (rosemary antioxidant extract from Flavex 027.020) was also used for comparative purposes.

\subsection{Cell culture}

Colorectal cancer cell lines, DLD1, and SW620, were obtained from the American Type Culture Collection (Manassas, VA, USA) and were cultured in DMEM media supplemented with $10 \%$ fetal bovine serum (LONZA Iberica, S.A, Barcelona, Spain) in an incubator with $95 \%$ humidity and $5 \% \mathrm{CO}_{2}$.

\subsection{Cell Viability}

The cytotoxic and antiproliferative activities of the different extracts in human CRC cell lines were determined by the MTT assay. Briefly, cells in the exponential growth phase were plated in 96-multiwell plates. After $24 \mathrm{~h}$, media was replaced with $200 \mu \mathrm{L}$ media containing serial concentrations of each extract (dissolved in DMSO) for $48 \mathrm{~h}$. The number of viable cells was determined at time zero (control growth wells) and after treatments. To determine the number of viable cells, tetrazolium MTT salt solution (Sigma) $(5 \mathrm{mg} / \mathrm{mL}$ in phosphate-buffered saline) was added for $3 \mathrm{~h}$. Then, the formazan produced in each well was solubilized by adding $200 \mu \mathrm{L}$ DMSO and measured using a spectrophotometer reader $(\lambda=560 \mathrm{~nm}$ ) (Biochrom Asys UVM 340 Microplate Reader; ISOGEN). Parameters for $50 \%$ of cell viability inhibition $\left(\mathrm{IC}_{50}\right)$ were calculated accordingly to $\mathrm{NIH}$ definitions using a logistic regression and are indicated in Figure 1 [54].

The synergism between 5-FU and FE or HFE extracts was analyzed by the combination index (CI) obtained using the Calcusyn software (version 2.1, Biosoft, Cambridge, UK), based on the Chou-Talalay method [55].

4.4. Extracellular flux Analysis of the Oxygen Consumption Rate (OCR) and Extracellular Acidification Rate (ECAR): MitoStress and GlycoStress Tests

Mitochondrial oxidative phosphorylation (Cell MitoStress Test) and aerobic glycolysis (Cell GlycoStress Test) were monitored with the XFe96 Cell Bionalyzer (Seahorse Biosciences, XFe96). Optimal cell density and drugs tritation were previously determined.

The dependency of the cells for aerobic glycolysis and oxidative phosphorylation were monitored after the injection of several modulators of both bioenergetic pathways.

Prior to the experiments, cells were pre-treated with different doses of FE and HFE $\left(1 / 2 \times \mathrm{IC}_{50}\right.$ and $\left.1 \times \mathrm{IC}_{50}\right)$ for $48 \mathrm{~h}$. Non-treated cells were kept as controls.

For MitoStress assay, 25,000 DLD1 or 40,000 SW620 cells were plated in a XFe-96 well plate and cells were kept for $6 \mathrm{~h}$ in DMEM 10\% FBS to allow the cells to attach. Then, medium was changed to $10 \mathrm{mM}$ glucose, $2 \mathrm{mM}$ glutamine, and $1 \mathrm{mM}$ pyruvate XFe DMEM (5mM HEPES), and cells were incubated for $1 \mathrm{~h}$ at $37^{\circ} \mathrm{C}$ without $\mathrm{CO}_{2}$. Three different modulators of the mitochondrial respiration were sequentially injected. After basal OCR determination (1 to 3 measurements), oligomycin $(1.5 \mu \mathrm{M})$, which inhibits ATPase, was injected to determine the amount of oxygen dedicated to ATP production by mitochondria, ( 3 to 6 measurements). To determine the maximal respiration rate (MRR) or Spare Respiratory Capacity, FCCP (carbonyl cyanide-4-(trifluoromethoxy) phenylhydrazone) was injected $\left(0.8 \mu \mathrm{M}\right.$ for DLD1 or $0.5 \mu \mathrm{M}$ for SW620) to free the gradient of $\mathrm{H}^{+}$from the mitochondrial intermembrane space (7 to 9 measurements), and thus, to activate maximal respiration. Finally, antimycin A and rotenone $(0.5 \mu \mathrm{M})$ were added to completely inhibit mitochondrial respiration (10 to 12 measurements).

GlycoStress assay: First, 20,000 DLD1 cells or 30,000 SW620 cells were plated in a XFe 96-well plate and kept for $6 \mathrm{~h}$ in DMEM 10\% FBS to allow the cells to attach. Then, culture medium was changed to $0.2 \mathrm{mM}$ glutamine XFe DMEM (5mM HEPES) to starve the cells for $1 \mathrm{~h}$. First, basal ECAR 
was measured (1 to 3 measurements). Then, $10 \mathrm{mM}$ glucose was injected (4 to 6 measurements) to the cells to determine glycolysis (this is the increased ECAR from the basal starved situation after glucose addition) and calculate the cells' capacity to use glucose. Next, maximal glycolytic capacity was monitored (7 to 9 measurements) after an injection of oligomycin, which inhibits the ATP production from the oxidative mitochondrial respiration. Finally, a third injection with $50 \mathrm{mM}$ DG was done to specifically shut down aerobic glycolysis (10 to 12 measurements).

\subsection{Measurement of Intracellular ATP Content}

For the quantification of the ATP content, the ATP-based assay CellTiter-Glo Luminescent Cell Viability kit was used (Promega, Madison, WI, USA; Cat \# G7571) following manufacturer's recommendations. Briefly, DLD1 or SW620 cells were first pre-treated (48h) with FE or HFE at $1 / 2 \times \mathrm{IC}_{50}, 1 \times \mathrm{IC}_{50}$ and $2 \times \mathrm{IC}_{50}$. Non-treated cells were kept as controls. A total of 7500 DLD1 cells or 15,000 SW620 cells were plated in 96-well clear bottom black polystyrene plates. Then, cells were maintained for $6 \mathrm{~h}$ in complete media, without treatments, to allow for attachment. Then, an equal volume of the single-one-step reagent provided by the kit was added to each well and rocked for $15 \mathrm{~min}$ at room temperature. Cellular ATP content was measured by a luminescent plate reader.

\subsection{Quantitative Real-Time Polymerase Chain Reaction (qRTPCR)}

DLD1 and SW620 cells $\left(0.35 \times 10^{6}\right.$ cells $)$ were treated with FE or HFE for 48 hours at different doses: $1 / 2 \mathrm{IC}_{50}, 1 \times \mathrm{IC}_{50}, 2 \times \mathrm{IC}_{50}$. Non-treated cells were kept as controls. For comparison and specificity of the targets, supercritical rosemary extract, whose molecular targets and mechanism of action has been previously characterised, was used (RE) [7,50].

Total RNA was extracted with Tri Reagent (Sigma). Then, $1 \mu \mathrm{g}$ of RNA was reverse-transcribed with High Capacity RNA to cDNA Master Mix system (Life Technologies, Carlsbad, CA, USA). qPCR was performed in the 7900HT Real-Time PCR System (Life Technologies) using VeriQuest SYBR Green qPCR Master Mix (Affymetrix, Santa Clara, CA, USA). Supplementary material shows the list and sequences of the oligos used and Taqman Probes used in this study. The $2^{-\Delta \Delta \mathrm{Ct}}$ method was applied to calculate the relative gene expression [56].

\subsection{Quantification of the Intracellular Neutral Lipid Content}

Intracellular neutral lipid content was measured using Oil Red O staining as previously described by Ramírez-Zacarías et al. [57]. Briefly, the cells were washed gently twice with ice-cold PBS (pH 7.4) and fixed with $10 \%$ formalin at room temperature for at least $30 \mathrm{~min}$ to $1 \mathrm{~h}$ in room temperature. After removal of the $10 \%$ formalin, wells were washed with $60 \%$ isopropyl alcohol for 5 min and then washed exhaustively with PBS. Wells were allowed to dry completely before the addition of filtered Oil Red O solution for $30 \mathrm{~min}$ at room temperature. Stained oil droplets were extracted with $100 \%$ isopropanol for $10 \mathrm{~min}$ to quantify intracellular lipids, and absorbance was measured spectrophotometrically at $510 \mathrm{~nm}$.

\subsection{Statistical analysis}

Results were analysed by ANOVA non-parametric with Bonferroni post hoc tests. Data were represented as mean \pm S.E.M of at least three independent experiments. Statistical differences were defined as $p<0.05\left(^{*}\right) ; p<0.01\left(^{* *}\right) ; p<0.005\left(^{* * *}\right) ; p<0.001\left(^{* * *}\right)$. Statistical analysis was performed with Graph Pad Prism 8 statistical software (Version 8.0.0, GraphPad Software, San Diego, CA, USA).

\section{Conclusions}

As summary, extracts from fenugreek, but not from quinoa, show strong inhibitory effects on the viability of CRC cell lines. Additionally, the cytotoxicity of the saponin-rich extract from fenugreek is superior than its acid-hydrolyzed form, which is probably due to synergisms with other bioactive 
compounds contained in the extract. For the first time, it has been demonstrated that the cytotoxic effects of these extracts on CRC cells may be related to alterations in the energetic metabolism of the cells, specifically to a reduction in their mitochondrial oxidative phosphorylation and to the inhibition of their aerobic glycolysis. Lastly, saponin and sapogenin-rich extracts from fenugreek have proven to target in a different manner the molecular pathways related to lipid metabolism in both CRC cell lines studied. This outcome may have important implications on their potential against CRC in the context of a precision nutrition, as the saponin-rich extract from fenugreek would be useful for the upregulation of genes promoting systemic anabolic effects in patients developing cachexia, whereas the sapogenin-rich extract would be locally useful to inhibit lipid targets implicated in a poorer prognosis in CRC cells. In this sense, this study shows the relevance of considering the coexisting compounds of the extracts or their hydrolysis transformation as innovative strategies to augment the clinical therapeutic potential of the extracts, and the specific subgroup of patients where each extract would be more beneficial in the frame of precision nutrition.

Supplementary Materials: The following are available online at http://www.mdpi.com/2072-6694/12/11/3399/s1. Figure S1: Quinoa extracts and cell bioenergetics; Figure S2: ATP content; Figure S3: 5-FU synergism; Figure S4: Red Oil Staining; List of oligos and probes used.

Author Contributions: Conceptualization: M.G.d.C., J.N.d.H., D.M., G.R. and A.R.d.M.; Methodology: M.G.d.C., J.N.d.H., M.R., S.W., A.B., A.Q.-F.; Formal analysis: M.G.d.C., J.N.d.H.; Writing-original draft preparation: M.G.d.C., J.N.d.H., D.M.; Supervision: M.G.d.C., J.N.d.H., D.M., G.R., A.R.d.M. All authors have read and agreed to the published version of the manuscript.

Funding: This research was funded by Ministerio de Economía y Competitividad, Spain (AGL2016-76736-C3-1-R) and Ministerio de Ciencia e Innovación, Spain (Plan Nacional I + D + I PID2019-110183RB-C21); Regional Government of Community of Madrid (P2018/BAA-4343-ALIBIRD2020-CM); Ramon Areces Foundation; EU Structural Funds and COST Action (CA17118). Sonia Wagner, Marina Reguero and Adrián Bouzas are predoctoral fellows from the industrial predoctoral program of Community of Madrid (IND2018/BIO-10097; IND2017/BIO-7826; IND2017/BIO-7857, respectively).

Acknowledgments: Joaquín Navarro del Hierro thanks the Ministerio de Educación, Cultura y Deporte for funding his research with a FPU predoctoral contract (FPU 15/04236).

Conflicts of Interest: The authors declare no conflict of interest.

\section{References}

1. Hanahan, D.; Weinberg, R.A. Hallmarks of cancer: The next generation. Cell 2011, 144, 646-674. [CrossRef] [PubMed]

2. Luo, X.; Cheng, C.; Tan, Z.; Li, N.; Tang, M.; Yang, L.; Cao, Y. Emerging roles of lipid metabolism in cancer metastasis. Mol. Cancer 2017, 16, 76. [CrossRef] [PubMed]

3. Sánchez-Martínez, R.; Cruz-Gil, S.; de Cedrón, M.G.; Álvarez-Fernández, M.; Vargas, T.; Molina, S.; García, B.; Herranz, J.; Moreno-Rubio, J.; Reglero, G.; et al. A link between lipid metabolism and epithelial-mesenchymal transition provides a target for colon cancer therapy. Oncotarget 2015, 6, 38719-38736. [CrossRef] [PubMed]

4. Aguirre-Portolés, C.; Feliu, J.; Reglero, G.; de Molina, A.R. ABCA1 overexpression worsens colorectal cancer prognosis by facilitating tumour growth and caveolin-1-dependent invasiveness, and these effects can be ameliorated using the BET inhibitor apabetalone. Mol. Oncol. 2018, 12, 1735-1752. [CrossRef]

5. Sparano, J.A.; Wang, M.; Zhao, F.; Stearns, V.; Martino, S.; Ligibel, J.A.; Perez, E.A.; Saphner, T.; Wolff, A.C.; Sledge, G.W.; et al. Obesity at diagnosis is associated with inferior outcomes in hormone receptor-positive operable breast cancer. Cancer 2012, 118, 5937-5946. [CrossRef]

6. Ramos-Lopez, O.; Milagro, F.I.; Allayee, H.; Chmurzynska, A.; Choi, M.S.; Curi, R.; De Caterina, R.; Ferguson, L.R.; Goni, L.; Kang, J.X.; et al. Guide for current nutrigenetic, nutrigenomic, and nutriepigenetic approaches for precision nutrition involving the prevention and management of chronic diseases associated with obesity. J. Nutrigenet. Nutrigenom. 2017, 10, 43-62. [CrossRef]

7. Gomez de Cedrón, M.; Laparra, J.M.; Loria-Kohen, V.; Molina, S.; Moreno-Rubio, J.; Montoya, J.J.; Torres, C.; Casado, E.; Reglero, G.; De Molina, A.R. Tolerability and safety of a nutritional supplement with potential as adjuvant in colorectal cancer therapy: A randomized trial in healthy volunteers. Nutrients 2019, 11, 2001. [CrossRef] 
8. Gómez de Cedrón, M.; Mouhid, L.; García-Carrascosa, E.; Fornari, T.; Reglero, G.; Ramírez de Molina, A. Marigold Supercritical Extract as Potential Co-adjuvant in Pancreatic Cancer: The Energetic Catastrophe Induced via BMP8B Ends Up With Autophagy-Induced Cell Death. Front. Bioeng. Biotechnol. 2020, 7, 455. [CrossRef]

9. Shiao, S.P.K.; Grayson, J.; Lie, A.; Yu, C.H. Personalized nutrition-Genes, diet, and related interactive parameters as predictors of cancer in multiethnic colorectal cancer families. Nutrients 2018, 10, 795. [CrossRef]

10. De la Iglesia, R.; Loria-Kohen, V.; Zulet, M.A.; Martinez, J.A.; Reglero, G.; de Molina, A.R. Dietary strategies implicated in the prevention and treatment of metabolic syndrome. Int. J. Mol. Sci. 2016, 17, 1877. [CrossRef]

11. Sung, J.; Ho, C.T.; Wang, Y. Preventive mechanism of bioactive dietary foods on obesity-related inflammation and diseases. Food Funct. 2018, 9, 6081-6095. [CrossRef] [PubMed]

12. Casas, R.; Estruch, R.; Sacanella, E. The Protective Effects of Extra Virgin Olive Oil on Immune-mediated Inflammatory Responses. Endocr. Metab. Immune Disord. Drug Targets 2017, 18. [CrossRef]

13. Kristo, A.S.; Klimis-Zacas, D.; Sikalidis, A.K. Protective role of dietary berries in cancer. Antioxidants 2016, 5, 37. [CrossRef] [PubMed]

14. Güçlü-Üstündağ, Ö.; Mazza, G. Saponins: Properties, applications and processing. Crit. Rev. Food Sci. Nutr. 2007, 47, 231-258. [CrossRef]

15. Singh, B.; Singh, J.P.; Singh, N.; Kaur, A. Saponins in pulses and their health promoting activities: A review. Food Chem. 2017, 233, 540-549. [CrossRef] [PubMed]

16. Navarro del Hierro, J.; Herrera, T.; Fornari, T.; Reglero, G.; Martin, D. The gastrointestinal behavior of saponins and its significance for their bioavailability and bioactivities. J. Funct. Foods 2018, 40, 484-497. [CrossRef]

17. Navarro del Hierro, J.; Cueva, C.; Tamargo, A.; Núñez-Gómez, E.; Moreno-Arribas, M.V.; Reglero, G.; Martin, D. In Vitro Colonic Fermentation of Saponin-Rich Extracts from Quinoa, Lentil, and Fenugreek. Effect on Sapogenins Yield and Human Gut Microbiota. J. Agric. Food Chem. 2020, 68, 106-116. [CrossRef] [PubMed]

18. Wang, J.j; Liu, Y.x.; Wen, D.; Yu, H.s.; Kang, L.p.; Pang, X.; Zhao, Y.; Ma, B.p.; Chen, Y.d. Study on steroidal saponins from Dioscorea zingiberensis and their platelet aggregation activities. Zhongguo Zhong Yao Za Zhi 2014, 39, 3782-3787. [CrossRef]

19. Chen, Y.; Tang, Y.M.; Yu, S.L.; Han, Y.W.; Kou, J.P.; Liu, B.L.; Yu, B.Y. Advances in the pharmacological activities and mechanisms of diosgenin. Chin. J. Nat. Med. 2015, 13, 578-587. [CrossRef]

20. Žiberna, L.; Šamec, D.; Mocan, A.; Nabavi, S.F.; Bishayee, A.; Farooqi, A.A.; Sureda, A.; Nabavi, S.M. Oleanolic acid alters multiple cell signaling pathways: Implication in cancer prevention and therapy. Int. J. Mol. Sci. 2017, 18, 643. [CrossRef]

21. Sen, A. Prophylactic and therapeutic roles of oleanolic acid and its derivatives in several diseases. World J. Clin. Cases 2020, 8, 1767-1792. [CrossRef] [PubMed]

22. Navarro del Hierro, J.; Reglero, G.; Martin, D. Chemical Characterization and Bioaccessibility of Bioactive Compounds from Saponin-Rich Extracts and Their Acid-Hydrolysates Obtained from Fenugreek and Quinoa. Foods 2020, 9, 1159. [CrossRef] [PubMed]

23. Vargas, T.; Moreno-Rubio, J.; Herranz, J.; Cejas, P.; Molina, S.; González-Vallinas, M.; Mendiola, M.; Burgos, E.; Aguayo, C.; Custodio, A.B.; et al. ColoLipidGene: Signature of lipid metabolism-related genes to predict prognosis in stage-II colon cancer patients. Oncotarget 2015, 6, 7348-7363. [CrossRef] [PubMed]

24. Vargas, T.; Moreno-Rubio, J.; Herranz, J.; Cejas, P.; Molina, S.; González-Vallinas, M.; Ramos, R.; Burgos, E.; Aguayo, C.; Custodio, A.B.; et al. Genes associated with metabolic syndrome predict disease-free survival in stage II colorectal cancer patients. A novel link between metabolic dysregulation and colorectal cancer. Mol. Oncol. 2014, 8, 1469-1481. [CrossRef]

25. Calle, E.E.; Kaaks, R. Overweight, obesity and cancer: Epidemiological evidence and proposed mechanisms. Nat. Rev. Cancer 2004, 4, 579-591. [CrossRef]

26. Greten, F.R.; Grivennikov, S.I. Inflammation and Cancer: Triggers, Mechanisms, and Consequences. Immunity 2019, 51, 27-41. [CrossRef]

27. Grivennikov, S.I.; Greten, F.R.; Karin, M. Immunity, Inflammation, and Cancer. Cell 2010, 140, 883-899. [CrossRef] 
28. González-Vallinas, M.; Molina, S.; Vicente, G.; De La Cueva, A.; Vargas, T.; Santoyo, S.; García-Risco, M.R.; Fornari, T.; Reglero, G.; Ramírez De Molina, A. Antitumor effect of 5-fluorouracil is enhanced by rosemary extract in both drug sensitive and resistant colon cancer cells. Pharmacol. Res. 2013, 72, 61-68. [CrossRef]

29. Fernández, L.P.; Sánchez-Martínez, R.; Vargas, T.; Herranz, J.; Martín-Hernández, R.; Mendiola, M.; Hardisson, D.; Reglero, G.; Feliu, J.; Redondo, A.; et al. The role of glycosyltransferase enzyme GCNT3 in colon and ovarian cancer prognosis and chemoresistance. Sci. Rep. 2018, 8. [CrossRef]

30. Brown, K.F.; Rumgay, H.; Dunlop, C.; Ryan, M.; Quartly, F.; Cox, A.; Deas, A.; Elliss-Brookes, L.; Gavin, A.; Hounsome, L.; et al. The fraction of cancer attributable to modifiable risk factors in England, Wales, Scotland, Northern Ireland, and the United Kingdom in 2015. Br. J. Cancer 2018, 118, 1130-1141. [CrossRef]

31. Parkin, D.M.; Boyd, L.; Walker, L.C. The fraction of cancer attributable to lifestyle and environmental factors in the UK in 2010. Br. J. Cancer 2011, 105, S77-S81. [CrossRef] [PubMed]

32. Bueno, M.J.; Jimenez-Renard, V.; Samino, S.; Capellades, J.; Junza, A.; López-Rodríguez, M.L.; Garcia-Carceles, J.; Lopez-Fabuel, I.; Bolaños, J.P.; Chandel, N.S.; et al. Essentiality of fatty acid synthase in the 2D to anchorage-independent growth transition in transforming cells. Nat. Commun. 2019, 10, 5011-5018. [CrossRef] [PubMed]

33. Liu, Q.; Luo, Q.; Halim, A.; Song, G. Targeting lipid metabolism of cancer cells: A promising therapeutic strategy for cancer. Cancer Lett. 2017, 401, 39-45. [CrossRef] [PubMed]

34. Bray, F.; Ferlay, J.; Soerjomataram, I.; Siegel, R.L.; Torre, L.A.; Jemal, A. Global cancer statistics 2018: GLOBOCAN estimates of incidence and mortality worldwide for 36 cancers in 185 countries. CA. Cancer J. Clin. 2018, 68, 394-424. [CrossRef] [PubMed]

35. Peck, B.; Schulze, A. Lipid Metabolism at the Nexus of Diet and Tumor Microenvironment. Trends Cancer 2019, 5, 693-703. [CrossRef] [PubMed]

36. Kuzu, O.F.; Noory, M.A.; Robertson, G.P. The role of cholesterol in cancer. Cancer Res. 2016, 76, $2063-2070$. [CrossRef] [PubMed]

37. Chen, H.; Qin, S.; Wang, M.; Zhang, T.; Zhang, S. Association between cholesterol intake and pancreatic cancer risk: Evidence from a meta-analysis. Sci. Rep. 2015, 5, 8243. [CrossRef] [PubMed]

38. Garcia-Estevez, L.; Moreno-Bueno, G. Updating the role of obesity and cholesterol in breast cancer. Breast Cancer Res. 2019, 21, 35. [CrossRef]

39. Vargas, T.; Moreno-Rubio, J.; Herranz, J.; Cejas, P.; Molina, S.; Mendiola, M.; Burgos, E.; Custodio, A.B.; De Miguel, M.; Martín-Hernández, R.; et al. 3’UTR polymorphismin ACSL1 gene correlates with expression levels and poor clinical outcome in colon cancer patients. PLoS ONE 2016, 11, 168423. [CrossRef]

40. Jeyakumar, A.; Dissabandara, L.; Gopalan, V. A critical overview on the biological and molecular features of red and processed meat in colorectal carcinogenesis. J. Gastroenterol. 2017, 52, 407-418. [CrossRef]

41. Van Raay, T.; Allen-Vercoe, E. Microbial Interactions and Interventions in Colorectal Cancer. In Bugs as Drugs; American Society of Microbiology: Washington, DC, USA, 2017; Volume 5, pp. 101-130.

42. Chapkin, R.S.; McMurray, D.N.; Lupton, J.R. Colon cancer, fatty acids and anti-inflammatory compounds. Curr. Opin. Gastroenterol. 2007, 23, 48-54. [CrossRef] [PubMed]

43. Navarro del Hierro, J.; Casado-Hidalgo, G.; Reglero, G.; Martin, D. The hydrolysis of saponin-rich extracts from fenugreek and quinoa improves their pancreatic lipase inhibitory activity and hypocholesterolemic effect. Food Chem. 2021, 338, 128113. [CrossRef] [PubMed]

44. Storniolo, C.E.; Martínez-Hovelman, N.; Martínez-Huélamo, M.; Lamuela-Raventos, R.M.; Moreno, J.J. Extra Virgin Olive Oil Minor Compounds Modulate Mitogenic Action of Oleic Acid on Colon Cancer Cell Line. J. Agric. Food Chem. 2019, 67, 11420-11427. [CrossRef] [PubMed]

45. Min, H.Y.; Pei, H.; Hyun, S.Y.; Boo, H.J.; Jang, H.J.; Cho, J.; Kim, J.H.; Son, J.; Lee, H.Y. Potent anticancer effect of the natural steroidal saponin gracillin is produced by inhibiting glycolysis and oxidative phosphorylation-mediated bioenergetics. Cancers 2020, 12, 913. [CrossRef]

46. Min, H.Y.; Jang, H.J.; Park, K.H.; Hyun, S.Y.; Park, S.J.; Kim, J.H.; Son, J.; Kang, S.S.; Lee, H.Y. The natural compound gracillin exerts potent antitumor activity by targeting mitochondrial complex II. Cell Death Dis. 2019, 10, 1-18. [CrossRef]

47. Mohammad-Sadeghipour, M.; Mahmoodi, M.; Karimabad, M.N.; Mirzaei, M.R. Diosgenin and 4-hydroxyisoleucine from fenugreek are regulators of genes involved in lipid metabolism in the human colorectal cancer cell line SW480. Cell J. 2021, 22, 514-522. [CrossRef] 
48. Zhou, L.; Shi, Y.; Guo, R.; Liang, M.; Zhu, X.; Wang, C. Digital Gene-Expression Profiling Analysis of the Cholesterol-Lowering Effects of Alfalfa Saponin Extract on Laying Hens. PLoS ONE 2014, 9, e98578. [CrossRef]

49. Liang, X.p.; Zhang, D.q.; Chen, Y.y.; Guo, R.; Wang, J.; Wang, C.z.; Shi, Y.h. Effects of alfalfa saponin extract on mRNA expression of Ldlr, LXR $\alpha$, and FXR in BRL cells. J. Zhejiang Univ. Sci. B 2015, 16, 479-486. [CrossRef]

50. González-Vallinas, M.; Molina, S.; Vicente, G.; Zarza, V.; Martín-Hernández, R.; García-Risco, M.R.; Fornari, T.; Reglero, G.; de Molina, A.R. Expression of MicroRNA-15b and the Glycosyltransferase GCNT3 Correlates with Antitumor Efficacy of Rosemary Diterpenes in Colon and Pancreatic Cancer. PLoS ONE 2014, 9, e98556. [CrossRef]

51. Navarro del Hierro, J.; Piazzini, V.; Reglero, G.; Martin, D.; Bergonzi, M.C. In Vitro Permeability of Saponins and Sapogenins from Seed Extracts by the Parallel Artificial Membrane Permeability Assay: Effect of in Vitro Gastrointestinal Digestion. J. Agric. Food Chem. 2020, 68, 1297-1305. [CrossRef]

52. Arpaia, N.; Campbell, C.; Fan, X.; Dikiy, S.; Van Der Veeken, J.; Deroos, P.; Liu, H.; Cross, J.R.; Pfeffer, K.; Coffer, P.J.; et al. Metabolites produced by commensal bacteria promote peripheral regulatory T-cell generation. Nature 2013, 504, 451-455. [CrossRef] [PubMed]

53. Michaudel, C.; Sokol, H. The Gut Microbiota at the Service of Immunometabolism. Cell Metab. 2020, 32, 514-523. [CrossRef] [PubMed]

54. Monks, A.; Scudiero, D.; Skehan, P.; Shoemaker, R.; Paull, K.; Vistica, D.; Hose, C.; Langley, J.; Cronise, P.; Vaigro-wolff, A.; et al. Feasibility of a high-flux anticancer drug screen using a diverse panel of cultured human tumor cell lines. J. Natl. Cancer Inst. 1991, 83, 757-766. [CrossRef] [PubMed]

55. Chou, T.C.; Talalay, P. Quantitative analysis of dose-effect relationships: The combined effects of multiple drugs or enzyme inhibitors. Adv. Enzym. Regul. 1984, 22, 27-55. [CrossRef]

56. Livak, K.J.; Schmittgen, T.D. Analysis of relative gene expression data using real-time quantitative PCR and the 2- $\Delta \Delta \mathrm{CT}$ method. Methods 2001, 25, 402-408. [CrossRef] [PubMed]

57. Ramírez-Zacarías, J.L.; Castro-Muñozledo, F.; Kuri-Harcuch, W. Quantitation of adipose conversion and triglycerides by staining intracytoplasmic lipids with oil red O. Histochemistry 1992, 97, 493-497. [CrossRef] [PubMed]

Publisher's Note: MDPI stays neutral with regard to jurisdictional claims in published maps and institutional affiliations.

(C) 2020 by the authors. Licensee MDPI, Basel, Switzerland. This article is an open access article distributed under the terms and conditions of the Creative Commons Attribution (CC BY) license (http://creativecommons.org/licenses/by/4.0/). 